Ann. Génét. Sél. anim., I973, 5 (2), I77-I88.

\title{
CONSANGUINITÉ ET PARENTÉ CHEZ LE PORC DE PIETRAIN
}

\author{
R. HANSET
}

Faculté de Médecine vétérinaire, Cureghem - Bruxelles

\section{RÉSUMÉ}

L'évolution du coefficient de consanguinité est étudiée, chez le porc de Piétrain, depuis son origine officielle c'est-à-dire depuis l'année 1950. Élevée au début (I5 P. Ioo en 195I), la valeur du coefficient de consanguinité n'est plus que de 5,2 p. Ioo en I96o.

Sur les pedigrees limités à cinq générations, d'animaux représentatifs de l'élevage d'élite, les coefficients de consanguinité moyens pour les années 1966, I970 et 1972 ont été respectivement de I, 26, I,59 et I,94 p. Ioo alors que, chez le Landrace belge, les coefficients de consanguinité moyen pour les années I 966 et 1970 ont été respectivement de 3, I I et 3,28 p. Ioo.

\section{I. - INTRODUCTION}

Il y a une vingtaine d'années que le porc de Piétrain a acquis, en Belgique, la qualité de race nationale, sinon en droit, tout au moins en fait. En effet, si, le I2 mai I950, est constitué, à Piétrain, un Syndicat des Éleveurs de Porcs, ce ne sera que dans le courant de I 956 que la reconnaissance officielle de la race sera étendue à tout le territoire de la Belgique.

L'identification et l'inscription des animaux reproducteurs, au livre généalogique ont constitué une des tâches principales du nouveau syndicat.

Le premier volume du Pig-Book du porc de Piétrain a été publié fin mars I95I.

La race était toutefois plus ancienne puisqu'on situe l'apparition de ses premiers représentants entre 1920 et 1930.

Il semble que le type ait été fixé assez tôt. Ceci s'explique si son déterminisme génétique est relativement simple, comme le suggèrent les travaux réalisés en France (OLIIVIER, I968). Une race noire, aux oreilles dressées, a contribué à sa constitution. Cette race est probablement le Berkshire anglais.

On peut concevoir que, grâce à des marqueurs génétiques spécifiques, on puisse, 
un jour prochain, identifier les races entrant dans la constitution du Piétrain. On n'aura pas pour autant éclairé l'origine du ou des gènes responsables de sa conformation particulière. En effet, on peut affirmer que des races entrant dans la constitution du Piétrain, aucune n'avait, comme telle, une conformation qui puisse faire penser à celle du Piétrain.

Les deux robes, pie-noire et blanche, ont longtemps coexisté et le choix de la robe pie-noire comme standard de la race est postérieur à la création du syndicat, en I950.

Les chances de survie d'un gène dans une population sont fonction de la taille de cette population et de la valeur sélective du gène, dans le milieu particulier où vit cette population. Lorsqu'il s'agit d'animaux exploités à des fins économiques, les chances de survie d'une " nouveauté génétique » dépendront de son intérêt économique éventuel.

En Belgique, la demande pour un porc maigre existe déjà, avant 1930. La préférence qu'on lui accorde sera, à son maximum, en I950, époque de l'expansion du Piétrain, dans tout le pays.

En mars I953, une catégorie spéciale est introduite dans les mercuriales du marché le plus important du pays, celui d'Anderlecht. Elle est réservée, en fait, aux porcs de Piétrain et s'intitule : "Porcs extra de viande - hors marché ". $\mathrm{Au}$ moment où cette rubrique est introduite, les prix pour cette catégorie oscillent entre 26 et $30 \mathrm{~F}$ contre $\mathrm{I} 8$ à $\mathrm{I} 9,5$ pour les porcs de la catégorie " demi-gras".

C'est un mécanisme économique bien connu que cet étalement des prix des différentes qualités d'un même produit, lorsque le marché est à la baisse.

\section{II. - CONSANGUINITÉ ET PARENTÉ}

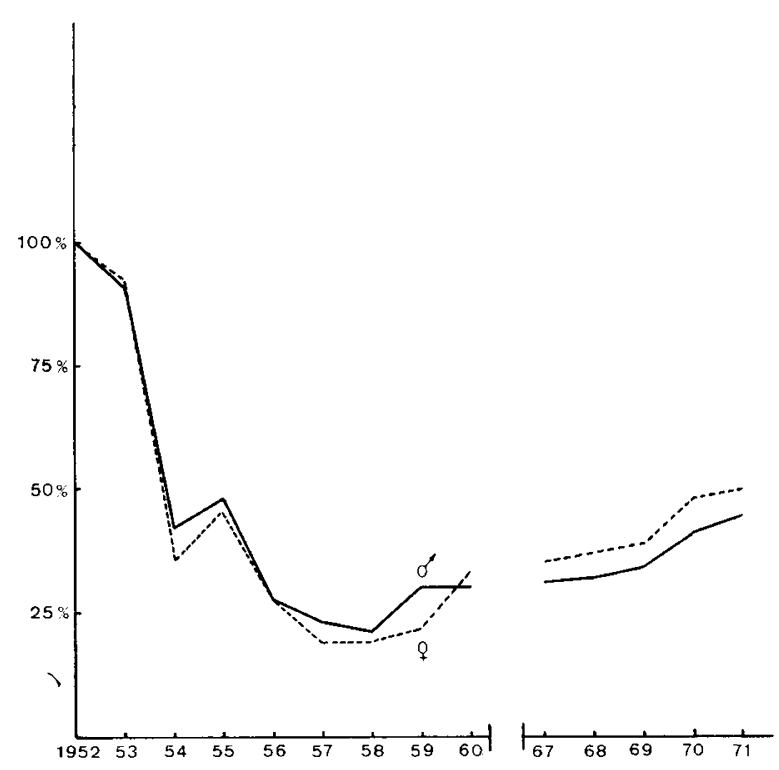

FIG. I. - Proportion des animaux inscrits au Pig-Book piétrain dans la province de Brabant 
L'extension rapide que connaît le porc de Piétrain se traduit dans le volume et la localisation géographique des inscriptions au Pig-Book.

Dans la figure I, on voit que la proportion des sujets inscrits au Pig-Book dans la province de Brabant, la province-mère, décrô̂t très vite. Jusqu'en I953, la quasitotalité de la race est localisée dans la province de Brabant ; quatre ans plus tard, cette province ne totalisera plus que $27 \mathrm{p}$. roo des inscriptions annuelles.

La fermeture du Pig-Book ne pouvait intervenir trop tôt afin que puisse se constituer une base de sélection suffisamment large. Une trentaine de verrats furent approuvés lors de la première expertise du 26 septembre I950. A partir de cette date, ne furent désormais inscrits au Pig-Book que des verrats d'origine connue.

On peut voir, dans le tableau $r$, que, dès I962, pratiquement tous les verrats inscrits au Pig-Book ont des parents connus. Pour les truies, ceci n'interviendra qu'à partir de I957, année de fermeture du Pig-Book.

\section{TABLEAU I}

Répartition des animaux inscrits au Pig-Book, selon que:

$\mathrm{I}^{\circ}$ leurs parents sont connus (PM) ; $2^{\circ}$ le père, seul, est connu (P?) ;

$3^{\circ}$ la mère, seule, est connue (? $\left.\mathrm{M}\right) ; 4^{\circ}$ le père et la mère ne sont pas connus (??)

\begin{tabular}{|c|c|c|c|c|c|c|c|c|c|c|c|c|}
\hline \multicolumn{7}{|c|}{ Mâles } & \multicolumn{6}{|c|}{ Femelles } \\
\hline & & $\begin{array}{c}\mathbf{P} \\
\mathbf{M}\end{array}$ & $\begin{array}{l}\mathrm{P} \\
?\end{array}$ & $\begin{array}{c}? \\
\text { M }\end{array}$ & $\begin{array}{l}? \\
?\end{array}$ & Tot. & & $\begin{array}{l}\mathrm{P} \\
\mathrm{M}\end{array}$ & $\begin{array}{l}\mathrm{P} \\
?\end{array}$ & $\stackrel{?}{\mathrm{M}}$ & $\begin{array}{l}? \\
?\end{array}$ & Tot. \\
\hline 1946 & & & & & 2 & 2 & & & 2 & & & 2 \\
\hline 1947 & & & & & 2 & 2 & 1 & $(7,69)$ & 7 & & 5 & 13 \\
\hline 1948 & 2 & $(20,22)$ & 6 & & 1 & 9 & 8 & $(10,39)$ & 32 & & 37 & 77 \\
\hline 1949 & 1 & $(5,88)$ & 16 & & & 17 & 52 & $(25,36)$ & 60 & 16 & 77 & 205 \\
\hline 1950 & 2 & $(28,57)$ & 5 & & & 7 & 45 & $(27,44)$ & 36 & 8 & 75 & 164 \\
\hline 1951 & 50 & $(65,79)$ & 9 & & 17 & 76 & 50 & $(70,42)$ & 17 & & 4 & 71 \\
\hline 1952 & 40 & $(100)$ & & & & 40 & 56 & $(72,73)$ & 8 & & 13 & 77 \\
\hline 1953 & 66 & $(97,06)$ & 1 & & 1 & 68 & 163 & $(38,53)$ & 5 & 2 & 253 & 423 \\
\hline 1954 & 155 & $(92,26)$ & 1 & & 12 & 168 & 354 & $(53,15)$ & 14 & 3 & 295 & 666 \\
\hline 1955 & 240 & $(100)$ & & & & 240 & 599 & $(56,09)$ & 4 & 9 & 456 & 1068 \\
\hline 1956 & 467 & $(98,73)$ & 4 & 1 & 1 & 473 & 1339 & $(63,52)$ & 16 & & 753 & 2108 \\
\hline 1957 & 338 & $(99,41)$ & & & 2 & 340 & 1687 & $(93,62)$ & 15 & & 100 & 1802 \\
\hline
\end{tabular}

$N . B$. : Entre parenthèses, le pourcentage d'individus de père et mère connus.

\section{A. - Matériel}

Pour le calcui du coefficient de consanguinité de Wright-Malécot (WRIGHT, I922; MALÉcot, I948), nous avons pris, pour les années étudiées, soit la totalité des sujets inscrits soit un échantillon au hasard. Les pedigrees aussi complets que possible, remontant jusqu'à l'origine officielle de sa race, ont été établis. Ils se rapportent à des sujets inscrits sous les millésimes $5 \mathrm{I}, 52,53$, 54 et 60 . En outre, les pedigrees complets ont également été établis pour un échantillon de reproducteurs ayant participé au Concours général de 1960.

D'autre part, les pedigrees, limités à cinq générations, d'animaux ayant participé aux Concours généraux de 1958 , I962, I966, I970 et 1972 pour le porc. de Piétrain, de 1958 , I962, I966 et $197^{\circ}$ pour le Landrace belge ont également été constitués et les coefficients de consanguinité calculés. 


\section{B. - Résultats}

Le tableau 2 donne les coefficients de consanguinité moyens, pour une succession d'échantillons annuels. Les valeurs données au tableau 2 ne représentent guère la consanguinité réelle. En effet, nous avons vu, tableau I, que trop d'animaux sont mentionnés comme " sans origine " alors que la plupart proviennent du même noyau d'élevage. C'est pourquoi nous avons calculé le coefficient de consanguinité moyen des individus pouvant théoriquement présenter une consanguiité c'est-à-dire des individus dont on connaît au moins le père et le grand-père maternel. On obtient alors les $\mathrm{F}$ moyens rapportés dans le tableau 3 .

TABLEAU 2

Consanguinité moyenne d'individus pris au hasard dans le Pig-Book

\begin{tabular}{|c|c|c|c|c|c|}
\hline $\begin{array}{l}\text { Millésime du no } \\
\text { de Pig-Book }\end{array}$ & Sexe & Effectif & $\begin{array}{c}\mathrm{F} \text { moyen } \\
(\%)\end{array}$ & Écart-type & $\begin{array}{c}\text { Valeurs } \\
\text { extrêmes } \\
(\%)\end{array}$ \\
\hline \multirow{2}{*}{1946 à 1950} & t & 35 & - & 一 & - \\
\hline & 우 & 443 & - & - & - \\
\hline \multirow{2}{*}{1951} & $\sigma$ & 32 & 5,86 & 10,88 & $0-37,5$ \\
\hline & 우 & 47 & 6,51 & 11,28 & $0-37,5$ \\
\hline \multirow{2}{*}{1952} & o & 40 & 5,35 & 7,49 & $0-25$ \\
\hline & 우 & 57 & 5,13 & 6,76 & $0-12,5$ \\
\hline \multirow{2}{*}{1953} & o & 41 & 4,02 & 8,15 & $0-37,5$ \\
\hline & 우 & 61 & 2,64 & 6,21 & $0-25$ \\
\hline \multirow{2}{*}{1954} & $\sigma$ & 47 & 3,21 & 7,83 & $0-40,62$ \\
\hline & 우 & 59 & 3,75 & 6,63 & $0-25$ \\
\hline \multirow{2}{*}{1960} & $\sigma$ & 46 & 4,94 & 4,69 & $0-16,43$ \\
\hline & 우 & 50 & 4,50 & 6,09 & $0-29,92$ \\
\hline \multirow{2}{*}{$1960^{*}$} & $\sigma$ & 39 & 3,04 & 4,75 & $0-28,57$ \\
\hline & q & 37 & 3,54 & 4,38 & $0-19,56$ \\
\hline
\end{tabular}

1960* : Animaux ayant participé au Concours général de 1960.

Au moment où s'ouvre le $P i g-B o o k$, il ne peut exister aucune consanguinité soit que les premiers animaux inscrits aient été déclarés " sans origine " soit qu'jls n'aient qu'une génération de parents connus. Toutefois, dès la génération suivante, 
une consanguinité relativement élevée apparaît (tabl. 3). C'était inévitable étant donné la taille de la population. Sur les 32 verrats inscrits au tome I du Pig-Book (millésimes 46 à 50), I2 sont fils et $\mathrm{I} 3$ sont petits-fils d'un même verrat Max 7 B I. Le même tome I du Pig-Book, dans sa partie réservée aux truies, compte 443 entrées. Pour les 229 d'entre elles dont le père est connu, 69 sont filles, 5r petites-filles et 26 arrière petites-filles de $\operatorname{Max} 7 \mathrm{~B} \mathrm{I}$.

\section{TABLEAU 3}

Consanguinité moyenne d'individus pris au hasard, dont le père et le grand-père maternel, au moins, sont connus

\begin{tabular}{|c|c|c|c|c|}
\hline $\begin{array}{l}\text { Millésime du no } \\
\text { de pig-book }\end{array}$ & Sexe & Effectif & $\mathrm{F}$ moyen $(\%)$ & Écart-type \\
\hline \multirow{2}{*}{1951} & $0^{x}$ & 12 & 15,62 & 12,92 \\
\hline & 우 & 21 & 14,58 & 13,01 \\
\hline \multirow{2}{*}{1952} & $\sigma$ & 29 & 6,52 & 7,26 \\
\hline & 오 & 35 & 8,35 & 6,90 \\
\hline \multirow{2}{*}{1953} & $\sigma$ & 20 & 8,24 & 10,15 \\
\hline & $q$ & 24 & 6,7 & 8,50 \\
\hline \multirow{2}{*}{1954} & 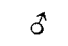 & 19 & 8,61 & 11,51 \\
\hline & 우 & 25 & 8,84 & 7,70 \\
\hline \multirow{2}{*}{1960} & $\delta$ & 43 & 5,28 & 4,65 \\
\hline & $q$ & 44 & $5,12 \cdots$ & 6,25 \\
\hline
\end{tabular}

Le rôle joué lors de la "naissance officielle " de la race par ce verrat, 7 B I et un de ses fils, Robert 48 B 3, ressort des données figurant aux tableaux 4 et 5 .

Au tableau 4, on peut trouver la parenté moyenne (coefficient de parenté, $R x y$, de Sewall Wright) des individus étudiés plus haut, à un certain nombre d'ancêtres. N'ont été retenus, pour le calcul de cette parenté moyenne, que les sujets dont on connaissait, au moins, le père.

La parenté moyenne à Max et à son fils, Robert, est élevée (de 40 à $50 \mathrm{p}$. Ioo pour les deux). Cette parenté demeure élevée jusqu'en r96o, époque à laquelle apparaissent de nouveaux géniteurs remarquables tels que : Clip de Pont (53 B II), son fils Émile $(54 \mathrm{~B}$ I25) et Gus (53 B I7). La parenté de Clip à Max est de $4 \mathrm{I}, 68$ p. Ioo.

Au tableau 5, on a calculé la contribution, à la consanguinité moyenne, de quelques ancêtres remarquables. Comme on pouvait le prévoir, les verrats Max et Robert sont les responsables de la quasi-totalité de la consanguinité observée, au 
TABLEAU 4

Parenté moyenne à certains ancêtres communs, de sujets dont le père, au moins, est connu (\%)

\begin{tabular}{|c|c|c|c|c|c|c|c|c|}
\hline Millésimes & Sexe & $\begin{array}{l}5 \text { B } 1 \\
\text { César }\end{array}$ & $\begin{array}{l}7 \mathrm{~B} 1 \\
\operatorname{Max}\end{array}$ & $\begin{array}{l}8 \mathrm{~B} 3 \\
\text { Robert }\end{array}$ & $\begin{array}{l}8 \mathrm{~B} 68 \\
\text { Mouche }\end{array}$ & $\begin{array}{l}53 \text { B } 11 \\
\text { Clip }\end{array}$ & $\begin{array}{c}53 \text { B } 17 \\
\text { Gus }\end{array}$ & $\begin{array}{c}54 \text { B } 125 \\
\text { Émile }\end{array}$ \\
\hline \multirow{2}{*}{1946 à 1950} & $0^{\star}$ & 7,03 & 28,90 & 17,19 & & & & \\
\hline & 우 & 3,38 & 22,05 & 13,97 & & & & \\
\hline \multirow{2}{*}{1951} & के & 7,03 & 27,93 & 9,76 & & & & \\
\hline & $q$ & 5,62 & 28,75 & 16,25 & & & & \\
\hline \multirow{2}{*}{1952} & t & 9,69 & 25,00 & 13,75 & & & & \\
\hline & 우 & 5,00 & 19,37 & 6,25 & & & & \\
\hline 1953 & $a+q$ & 2,91 & 17,71 & 9,00 & 5,2 & & & \\
\hline 1954 & $\hat{o}+q$ & & 17,85 & 15,95 & 2,71 & & & \\
\hline 1960 & $\pi+q$ & 2,29 & 12,58 & 9,43 & 4,42 & 14,52 & 6,56 & 11,82 \\
\hline $1960^{*}$ & $a+q$ & 2,29 & 10,41 & 7,87 & 3,69 & 11,39 & 7,69 & 6,29 \\
\hline
\end{tabular}

1960* : Animaux ayant participé au Concours général de 1960.

\section{TABLEAU 5}

Participation à la consanguinité moyenne de quelques ancêtres remarquables (en \%)

\begin{tabular}{|c|c|c|c|c|c|c|c|c|}
\hline Millésimes & Sexe & $\begin{array}{l}5 \text { B } 1 \\
\text { César }\end{array}$ & $\begin{array}{l}7 \text { B } 1 \\
\operatorname{Max}\end{array}$ & $\begin{array}{c}8 \text { B 3 } \\
\text { Robert }\end{array}$ & $\begin{array}{l}8 \text { B } 68 \\
\text { Mouche }\end{array}$ & $\begin{array}{c}53 \text { B } 11 \\
\text { Clip }\end{array}$ & $\begin{array}{c}53 \text { B } 17 \\
\text { Gus }\end{array}$ & $\begin{array}{c}54 \text { B } 125 \\
\text { Émile }\end{array}$ \\
\hline \multirow{2}{*}{1951} & a & 7,14 & 75 & & & & & \\
\hline & 우 & & 100 & & & & & \\
\hline \multirow{2}{*}{1952} & $\sigma^{*}$ & 8,76 & 53,28 & 23,36 & & & & \\
\hline & 우 & 33,33 & 66,66 & & & & & \\
\hline 1953 & $\delta+p$ & 0,48 & 64,03 & 16,31 & 9,59 & & & \\
\hline 1954 & $\tilde{d}+q$ & & 32,89 & 34,13 & 2,97 & & & \\
\hline 1960 & $0+q$ & $\begin{array}{l}0,18 \\
(3)\end{array}$ & $\begin{array}{c}12,83 \\
(20)\end{array}$ & $\begin{array}{l}9,20 \\
(12)\end{array}$ & $\begin{array}{l}1,19 \\
(6)\end{array}$ & $\begin{array}{c}18,00 \\
(27)\end{array}$ & $\begin{array}{c}2,94 \\
(19)\end{array}$ & $\begin{array}{c}15,80 \\
(27)\end{array}$ \\
\hline $1960^{*}$ & $0+q$ & $\begin{array}{l}0,48 \\
(4)\end{array}$ & $\begin{array}{c}15,26 \\
(17)\end{array}$ & $\begin{array}{l}8,80 \\
(16)\end{array}$ & $\begin{array}{l}1,01 \\
(8)\end{array}$ & $\begin{array}{l}22,87 \\
(21)\end{array}$ & $\begin{array}{l}8,54 \\
(24)\end{array}$ & $\begin{array}{l}1,88 \\
(8)\end{array}$ \\
\hline
\end{tabular}

1960* : Animaux ayant participé au Concours général de 1960.

Entre parenthèses, le nombre de descendants directs (fils ou filles) par lesquels l'ancêtre est représenté. 
début tout au moins. En I960, cette consanguinité due à Max et à Robert est devenue une consanguinité éloignée tandis que leurs descendants, Clip et Émile, sont responsables d'une consanguinité rapprochée. On trouvera également au tableau 5, aussi bien pour l'échantillon au hasard de Ig6o que pour l'échantillon d'animaux ayant participé au Concours général de I96o, et entre parenthèses, le nombre de descendants (fils ou filles) par lesquels les divers ancêtres communs sont représentés dans ces pedigrees.

\section{TABLEAU 6}

Consanguinité moyenne d'échantillons de quarante animaux (1) (vingt mâles, vingt femelles)

ayant participé aux Concours généraux de 1958, 1962, 1966, 1970, 1972

1. - Piétrain

\begin{tabular}{c|c|c|c|c}
\hline Anné & $n$ & F moyen (\%) & Écart-type & Valeurs extrêmes \\
& & & & \\
\hline & & & & \\
1958 & 40 & 2,35 & 3,45 & $0-13,28$ \\
1962 & 40 & 2,23 & 2,58 & $0-13,67$ \\
1966 & 40 & 1,26 & 2,36 & $0-12,89$ \\
1970 & 40 & 1,59 & 2,09 & $0-11,91$ \\
1972 & 40 & 1,94 & 3,22 & $0-14,84$ \\
\hline
\end{tabular}

II. - Landrace belge

\begin{tabular}{c|c|c|c|c}
\hline Année & $n$ & F moyen (\%) & Écart-type & $\begin{array}{c}\text { Valeurs extrêmes } \\
(\%)\end{array}$ \\
\hline & & & & \\
\hline 1958 & 40 & 1,17 & 2,96 & $0-12,50$ \\
1962 & 40 & 1,15 & 2,08 & $0-8,20$ \\
1966 & 40 & 3,11 & 2,75 & $0-9,76$ \\
1970 & 40 & 3,28 & 4,60 & $0-18,55$ \\
\hline
\end{tabular}

(1) Pedigres comportant cinq générations. Seuls ont été retenus les animaux dont le père et le grand-père maternel, au moins, sont connus.

En ce qui concerne le $\mathrm{F}$ moyen calculé sur des échantillons de quarante animaux ayant participé aux Concours généraux de 1958, I962, I966, I968, I970 et 1972, il est donné au tableau 6 . Les pedigrees de ces animaux ont été établis sur 5 générations. A partir de I966, ces pedigrees ne comptent pratiquement plus de manquants. On constate que la consanguinité rapprochée est, à présent, relativement faible, dans la race de Piétrain. Dans le même tableau, on donne, pour comparaison, le F moyen calculé sur des échantillons comparables de porcs Landrace belge. Pour cette race également, les pedigrees ne comptent pratiquement plus de manquants à partir de I966. 


\section{C. - Discussion}

Le Pig-Book n'ayant été fermé qu'en I957, pour les truies tout au moins, les pedigrees doivent nécessairement compter beaucoup de manquants. Ces manquants représentent soit des individus apparentés aux individus nommément présents dans les pedigrees soit des individus appartenant à une autre population ou éventuellement à une autre race.

Dans le premier cas, la consanguinité calculée sous-estime la consanguinité réelle, dans le second cas, il n'y a pas sous-estimation.

$\mathrm{Si}$ on considère un pedigree à cinq générations, un manquant respectivement en $5^{\mathrm{e}}, 4^{\mathrm{e}}, 3^{\mathrm{e}}, 2^{\mathrm{e}}$ génération réduira la consanguinité calculée à $\mathrm{I} / \mathrm{I} 6,7 / 8,3 / 4$, et $\mathrm{I} / 2$ de la consanguinité réelle.

Au début, on a inscrit au Pig-Book des sujets dont un parent était d'une autre race. Ainsi, dans le tome I du Pig-Book, au chapitre des truies, on trouve parfois la mention " père Grand Yorkshire ". Que de tels sujets croisés aient pu être inscrits comme satisfaisant aux critères de la race n'a rien d'étonnant. En effet, KNOERTZER (I96I) remarque que des produits du croisement Piétrain $\times$ Large White font penser à la conformation du type culard, certains ayant la fossette, au-dessus de la queue, si caractéristique du Piétrain. La robe blanche n'excluait pas l'inscription.

Les valeurs minimales que l'on peut assigner à la consanguinité moyenne du Piétrain sont celles du tableau 2. Les valeurs du tableau 3, pour avoir un sens, supposent : $I^{\circ}$ que les pedigrees où on connaît au moins le père et le grand-père maternel soient représentatifs de l'ensemble autrement dit que les pedigrees qui ne satisfont pas à ces conditions ne se rapportent pas à des produits de croisement; $2^{\circ}$ que les manquants dans les pedigrees retenus correspondent à des sujets extérieurs.

Si la première considération n'est pas satisfaite, les $\mathrm{F}$ seront moins élevés ; si la deuxième condition n'est pas satisfaite, les $\mathrm{F}$ seront plus élevés. Admettre une compensation entre ces deux effets contradictoires revient à considérer les valeurs de $\mathrm{F}$ du tableau 3 comme estimant valablement le coefficient de consanguinité du Piétrain, aux différentes époques envisagées.

Élevée au début, cette consanguinité va ensuite en diminuant. Théoriquement, dans une population fermée, la consanguinité ne peut diminuer. Néanmoins, une population cloisonnée en isolats verra sa consanguinité diminuer si des échanges ont lieu entre les isolats. De même, une population après être passée par un goulot d'étranglement (" bottleneck ») verra sa consanguinité diminuer si des sujets extérieurs sont introduits dans la population.

Dans le village même de Piétrain, du fait du nombre réduit de mâles (deux en tout, le père, Max et le fils, Robert), une consanguinité étroite est inévitable. Pour répondre à une demande qui dépasse l'offre, des croisements extérieurs auront lieu. En outre, on ne peut perdre de vue que des porcs du type Piétrain existaient ailleurs que dans le village de Piétrain. A leur tour, ils seront déversés dans le pool commun de la jeune race.

Il n'est pas sans intérêt de comparer les valeurs obtenues, chez le Piétrain, à celles obtenues dans d'autres races (tabl. 7).

Dans la première partie du tableau 7 , sont reproduites les valeurs de $F$ calculées sur des pedigrees remontant à l'origine de la race; dans la seconde partie, il s'agit de pedigrees limités à quelques générations (4 à 6). 
TABLEAU 7

Consanguinité observée dans quelques races porcines

A. - Consanquinité totale (calculé depuis l'origine)

\begin{tabular}{|c|c|c|}
\hline Races & $\mathrm{F}$ total $(\%)$ & Auteurs \\
\hline 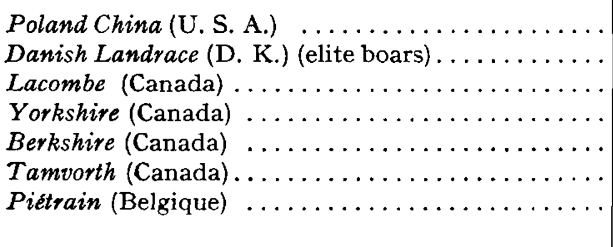 & $\begin{array}{c}9,8 \\
14,9 \\
12,5 \\
7,0 \\
7,0 \\
12,2 \\
5,2 \\
\text { (en } 1960 \text { ) }\end{array}$ & $\begin{array}{l}\text { LUSH et ANDERSON (1939) } \\
\text { JonsSON et JENSEN (1969) } \\
\text { FREDEEN et STOTHART (1969) } \\
\text { FrEDEEN } \text { et al. (1969) } \\
\quad-\quad-\quad- \\
- \text { Présente étude }\end{array}$ \\
\hline
\end{tabular}

B. - Consanguinité partielle (nombre limité de générations)

\begin{tabular}{|c|c|c|}
\hline Races & $\mathrm{F}$ total $(\%)$ & Auteurs \\
\hline 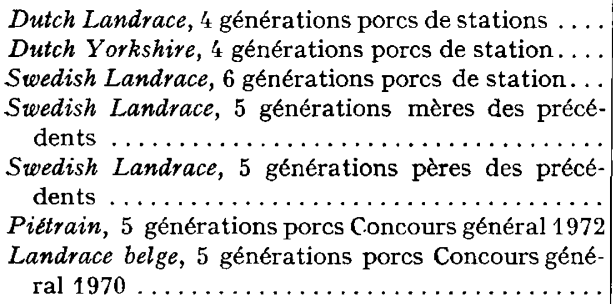 & $\begin{array}{l}1,66 \\
3,07 \\
202 \\
1,14 \\
1,55 \\
1,94 \\
3,28\end{array}$ & $\begin{array}{l}\text { BeKedam (1959) } \\
-\quad- \\
\text { BRÄNnang } \\
-\quad 1969) \\
\text { Présente étude } \\
\text { Présente étude }\end{array}$ \\
\hline
\end{tabular}

La consanguinité de la race de Piétrain n'est vraisemblablement pas plus élevée que celles des autres races. Rappelons que les nouvelles races américaines (Minnesota, Montana, Beltsville,...) avaient atteint un coefficient de consanguinité de l'ordre de $30 \mathrm{p}$. IOo au moment où elles furent reconnues (voir LASLEY, Ig63). Il est vrai que dans ce cas, la consanguinité est nulle à l'origine (mélange de deux ou trois races) et que, à partir de là, les pedigrees étaient complets.

L'étude de différents marqueurs génétiques pourrait nous éclairer davantage sur l'intensité de la consanguinité subie par la race de Piétrain. En effet, dans une petite population, les changements fortuits des fréquences géniques vont, en moyenne, engendrer une diminution nette de 1'hétérozygotie et un accroissement de 1'homozygotie (Crow et Krmura, r970). Le nombre d'allèles, à tout locus, aura tendance à diminer.

Des données obtenues par GruHN (1972) et citées par HaRING et KALM (I972) font apparaître que des allèles de certains systèmes (préalbumine, amylase) sont presque fixés dans la race de Piétrain, à la différence de ce qui est observé chez le Landrace belge. 
Néanmoins, la variabilité génétique, à l'intérieur de la race, pour les caractères économiques (engraissement et carcasse) ne paraît pas plus faible que dans les autres races (HANSET et VAN SNICK, I972).

D'autre part, des auteurs (KIRSCH et al., I963) attribuent à une consanguinité élevée le fait que les performances reproductrices et d'engraissement du Piétrain soient plus faibles que chez d'autres races européennes. Cette interprétation ne nous paraît pas correcte. En effet, cette infériorité étant, selon cette opinion, une manifestation du phénomène de dépression due à la consanguinité, elle ne devrait pas se trouver chez les produits de croisement.

Or, si on consulte les tableaux 8 et 9, on constate que cette infériorité du Piétrain se retrouve chez les produits de croisement, qu'il s'agisse de la fertilité (tab1. 8) ou des caractères d'engraissement (tabl. 9).

\section{TABLEAU 8}

Performances de truies hybrides comparées à celles de truies Large White

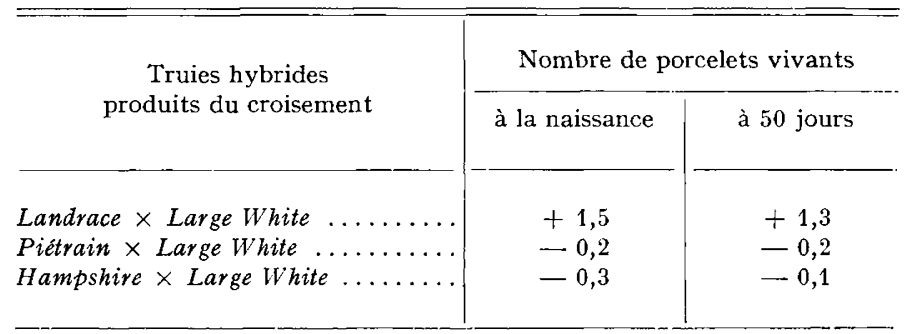

KING (1968) cité par Smith et Howard (1972).

TABLEAU 9

Performances des produits de croisements entre des verrats de Piétrain ou Landrace avec des truies Large White

\begin{tabular}{|c|c|c|c|}
\hline & $\begin{array}{c}\text { Piétrain } \\
\times \text { Large White }\end{array}$ & $\begin{array}{c}\text { Landrace } \\
\times \text { Large White }\end{array}$ & Différence \\
\hline Gain quot.moyen (g) & 680 & 750 & -70 \\
\hline Indice consomm. $\left(\mathrm{K}_{0}\right) \ldots$ & 3,95 & 3,83 & 0,12 \\
\hline Longueur $(\mathrm{cm}) \ldots \ldots \ldots$ & 83,0 & 86,6 & $-3,6$ \\
\hline Surface longis, dorsi $\left(\mathrm{cm}^{2}\right)$. & 38,2 & 30,4 & 7,8 \\
\hline P. 100 maigre carcasse $(\%)$.. & 45,4 & 43,2 & 2,2 \\
\hline
\end{tabular}

D'après King (1972) cité par Smith et Howard (1972).

On ne peut perdre de vue qu'il s'agit d'une race dont le format est inférieur à celui des races auxquelles on la compare généralement. A poids constant, la carcasse du Piétrain est plus courte. Le verrat Max 7 B I était d'ailleurs un sujet relativement 
court. Le format d'une race conditionne généralement sa croissance. Il pourrait conditionner également la taille des portées. Cela a été démontré chez la Souris (FALCONER, I955 ; LAND, I970), chez le Chien (KAISER, I972). Chez le Porc, cette relation est moins claire (LEGAULT, I970).

Lors d'une expérience de sélection en faveur d'un format réduit, chez le Porc, DetTTMERs et al. (I97I) observent une réduction de la taille de portée de un porcelet. Toutefois, cette réduction pouvait aussi bien être un effet de la consanguinité qu'un effet de la diminution $\mathbf{d u}$ format.

Des races qui ont vraisemblablement participé à la constitution du porc de Piétrain, il en est une, le Berkshire, qui n'est pas particulièrement prolifique.

D'autre part, il faut aussi se demander si certaines infériorités du Piétrain ne sont pas le corollaire de sa conformation particulière. On a, en effet, trouvé une corrélation négative tant phénotypique que génétique, entre le gain quotidien moyen et la proportion de morceaux maigres dans la carcasse (HANSET et VAN SNICK, r972).

Reçu pour publication en janvier 1973.

\section{REMERCIEMENTS}

L'auteur tient à adresser ses plus vifs remerciements à M. l'ingénieur R. CAMERLYNCK, Conseiller de Zootechnie de l'État, à M. LAPLANCHE, informaticien au Centre de Calcul et de Traitement de l'Information de l'Université de Liège, à M. Roesems, technicien au Ministère de l'Agriculture. Il remercie également M. L. Ollivier (I. N. R. A., Jouy en Josas) pour ses remarques et suggestions à la lecture du manuscrit.

\section{SUMMARY}

\section{INBREEDING AND KINSHIP IN PIÉTRAIN PIG}

The evolution of inbreeding in the Pietrain pig breed is studied since the official origin of the breed, in I950. High at the beginning ( 5 p. roo in the year I95I), a. 5, 2 p. I00 inbreeding was observed in I96o.

For pedigrees restricted to five generations, of animals representative of the elite, the average coefficients of inbreeding, in 1966,1970 and 1972 , were respectively $\mathrm{I}, 26 \mathrm{p}$. I00 I,59 p. I00, and I,94 P. Ioo while, for the Belgian Landrace, the average coefficients of inbreeding, in I966 and I970, were respectively : 3, I I and 3,28 p. I00. The relation between inbreeding, on one hand, fertility and rate of gain on the other hand, is discussed in the case of the Piétrain pig breed.

\section{RÉFÉRENCESS BIBLIOGRAPHIQUES}

Bekedam M., r959. Inbreeding in the Dutch Swine breeds. Z. Tierz. ZüchtBiol., 73, 2 I8-220. BräNANG E., I969. The inbreeding and selection depression in Swedish Landrace Pigs. Lantbr. Högsk. Annir, 35, 809-8I4.

Crow J. F., Kimura M., i97o. An Introduction to Population Genetics Theory. Harper and Row. New York. 
Dettmers A. E., Rempel W. E., Hacker D. E., I97I. Response to recurrent mass selection for small size in Swine. J. Anim. Sci., 33, I2I2-I 2 I5.

FALCONER D. S., I955. Patterns of response in selection experiments with mice. Cold Spring Harbor Symp. Quant. Biol. 20, 178-196.

Fredeen H. T., Stothart J. G., I969. Development of a new breed of pigs : the Lacombe. I. Foundation and development procedures. Can. J. Anim. Sci. 49, 237-246.

Fredeen H. T., Hickman C. G., Stothart J. G., r969. Inbreeding and relationship for three breeds of pigs in Canada. Can. J. Anim. Sci., 49, 275-289.

HANSET R., VAN SNICK C., I972. Les paramètres génétiques. des caractères d'engraissement et de carcasse chez le porc de Piétrain. Ann. Génét. Sél. anim., 4, 45r-467.

HaRING F., Kalm E., I972. Bestimmungsgrunde, Ergebnisse und Aussichten der Einführung der belgischen Schweinerassen in West Deuschland. Journée Internationale du Porc. Bruxelles.

Jonsson P., Jensen P., r969. Degree of inbreeding in Danish Landrace elite breeding. Forögslab Arb., Kbh. I969, 97-98.

KAISER G., r97I. Die Reproduktionsleistung der Haushunde in ihrer Beziehung zur Körpergrösse und zum Gewicht der Rassen. Z. Tierz. Züchtbiol. 88, 118, 241, 316.

Kirsch W., Fender M., Rabold K., Fewson D., Schoen P., r963. Vergleichende Zucht-, Mastund Ausschlachtungsversuche mit veredelten Landschweinen, Piétrain-Schweinen und $\mathrm{F}_{1}$-Kreuzungstieren. Züchtgskde, 35, 254-264.

Knoertzer E., I96I. Le croisement porcin Piétrain $\times$ Large White. Bull. Techn. Inf. Agric., 165, IO2I-I042.

LAND R. B., I970. Genetic and phenotypic relationships between ovulation rate and body weight in the mouse. Genet. Res., 15, I7 I-182.

LASLEy J. F., I963. Genetics of Livestock Improvement. Prentice-Hall, New Jersey.

LEGAULT C., 1970. Relation entre les performances de reproduction et les performances d'engraissement et de carcasse chez le porc. F.E.Z. Gödollö, r97o.

Lush J. L., Anderson A. L., 1939. A genetic history of Poland-China swine. J. Hered., 30 149-156, 219-224.

MALÉcot G., 1948. Les mathématiques de l'hérédité. Masson, Paris.

Ollivier L., I968. Étude du déterminisme héréditaire de l'hypertrophie musculaire du porc de $P i e ́$ train. Ann. Zootec., 17, 393-407.

Smith W. C., Howard A. N. 1972. Purebred and crossbred performance of the Piétrain breed in Britain. Journée Internationale du Porc. Bruxelles.

Wright S., I922. Coefficients of inbreeding and relationship. Amer. Nat. 56, 330-338. 